\title{
EVALUACIÓN DE UNA EXPERIENCIA DE AULA CON UN DISEÑO BASADO EN LA TEORÍA DE LA ELABORACIÓN
}

\author{
MACIAS, A., MATURANO, C.I. y CASTRO, J.I. \\ Instituto de Investigaciones en Educación en las Ciencias Experimentales (IIECE). \\ Facultad de Filosofia, Humanidades y Artes. Universidad Nacional de San Juan. \\ Av. José I. de La Roza, 230 Oeste. San Juan. C.P. 5400. República Argentina.
}

\section{SUMMARY}

This work proposes a model of a curricular design in Physics and its application in the classroom. It is developed according to the guidelines of the elaboration theory and it is put into practice in a basic university course.

It includes an analysis of the structure of knowledge and an organization of the contents involved at different levels. Besides, it provides information about what and how to teach and what and how to evaluate.

The results achieved in the classroom according to what is prescribed in this design about Waves and Sound are analyzed and discussed in this paper.

\section{INTRODUCCIÓN}

La problemática educativa en física nos enfrenta diariamente en el aula con dificultades incluidas dentro de un universo más complejo, que corresponden a la educación en general.

Esto lleva a investigadores en educación y a educadores a buscar soluciones con diferentes líneas de abordaje, que, en nuestro caso, están enfocadas al estudio del proceso de enseñanza-aprendizaje, en especial al diseño curricular de temas puntuales de física y su puesta en práctica en el aula.

En este caso nos hemos centrado en el análisis y la aplicación de la teoría de la elaboración a una unidad didáctica de Física le nivel básico universitario.

Los modelos de aprendizaje con una raíz profunda en los aportes de la psicología y el recurso de las modernas tecnologías en la enseñanza han servido de marco para esta propuesta, que está enmarcada dentro del modelo constructivista del aprendizaje escolar, al cual le corresponde, como consecuencia, un modelo curricular abierto. En tal sentido, hemos reinterpretado la teoría de la elaboración con la intención de hacer más accesible su uso práctico, dando un ejemplo concreto.

\section{MARCO TEÓRICO}

El trabajo se ha realizado desde un enfoque de investigación-acción; es decir, que se construye un diseño curricular mediante una actividad intelectual y creadora del equipo, donde se profundizan los conocimientos y luego se transfiere la experiencia al aula. Es indispensable planificar para que la intervención pedagógica se desarrolle dentro del modelo constructivista. 
Ángel Pérez Gómez (1989), César Coll (1987) y Rufina Gutiérrez (1992) acercan a los investigadores de habla castellana la teoría de la elaboración, como una importante teoría de la instrucción. Según estos autores, ésta es un modelo desarrollado principalmente por Reigeluth y Merrill, quienes asientan sus trabajos en dos corrientes principales: la de la psicología cognitiva y la de los modelos del aprendizaje basados en el procesamiento de la información.

Partimos de la propuesta de César Coll, que aconseja que, del conjunto de estrategias instruccionales que prescribe la teoría de la elaboración, es conveniente interesarse por las que se refieren a la organización y secuenciación de los contenidos de la enseñanza y la concreción con estrategias adecuadas para que el alumno logre un aprendizaje significativo.

En la teoría de la elaboración se tienen en cuenta los siguientes aspectos:

\section{- Organización y secuenciación de la enseñanza}

- Forma de guiar el aprendizaje

- Motivación de los alumnos

- Forma de ayudar a los alumnos a autoevaluarse

- Evaluación de los aprendizajes

- Corrección de las estrategias utilizadas según los resultados observados.

La propuesta de la teoría de la elaboración en lo que se refiere a la organización de la enseñanza consiste en una analogía del zoom, siendo éste el punto de partida. Es decir, que se debe comenzar dando una visión general del tema a tratar aunque en este primer momento no puedan apreciarse detalles. Luego, con el fin de obtener una información más minuciosa, debe centrarse la atención en un aspecto o parte de la organización general anteriormente presentada, volviendo periódicamente al plano general pará su ampliación.

A fin de analizar todas las partes involucradas, debe repetirse este proceso las veces que sea necesario. E1 enriquecimiento y nivel de detalle que pueden lograrse es ilimitado.

Es importante destacar que, según esta teoría, deben secuenciarse los contenidos apuntando al logro de diferentes niveles de procesamiento cognitivo, tanto de hechos como de conceptos, principios y procedimientos. El hincapié que se hace en la organización de los contenidos no resta importancia a otros aspectos contemplados en el proceso de enseñanza-aprendizaje, como son la motivación del alumno y la evaluación.

En resumen, la propuesta consiste en presentarle al alumno los contenidos organizados de lo más general a lo más pormenorizado y de lo más simple a lo más complejo.
Esta teoría de la instrucción permite, según expresa César Coll:

- presentar el contenido a enseñar en términos muy generales o simples;

- introducir el nivel de complejidad que se pretende en cada uno de los componentes.

Analizaremos a continuación los pasos a seguir de acuerdo con lo propuesto por la teoría de la elaboración, utilizando como bibliografía de referencia los textos de Alvarenga y Máximo (1983), Gettys y otros (1991), Resnick y Halliday (1980) y Sears y otros (1988).

\section{DISEÑO CURRICULAR ENMARCADO EN LA TEORIA DE LA ELABORACION}

\section{Epítome}

Según Coll (1987), el primer paso de la secuencia recibe el nombre de epitome, el cual se diferencia de un resumen porque articula los componentes de un contenido en vez de sintetizarlas.

Esta integración de los componentes destaca lo fundamental de lo accesorio, lo detallado y lo complejo. Estas características del epítome no indican que queden excluidos de él ejemplos prácticos y pruebas experimentales, pues ellos son los que permiten hacer que el alumno realice aprendizajes significativos.

Debemos, entonces, definir el epítome inicial como expresión integradora de los contenidos a desarrollar en la unidad seleccionada; en nuestro caso, "Ondas y Acústica», como figura en el cuadro I:

\section{Cuadro I}

Vivimos rodeados de ondas.

Para comprobarlo basta que mircmos el mar o la superficie de un lago.

Vemos la televisión, escuchanos la ridio, nos comunicamos tclefónicamente o por fax utilizando fenómenos ondulatsrios.

Los terremotos, las cuerdas de los instrumentos masicules y el horno de microondas son ejemplos, entre otros, de propagaciones ondulatorias.

\section{Mapas conceptuales}

$\mathrm{Al}$ diseñar debemos elaborar los mapas conceptuales partiendo de los conceptos generales y avanzando en forma progresiva hasta incluir los más específicos.

La teoría de las ondas, en su forma más general, es esencialmente una teoría matemática, cuyo contenido conceptual está determinado por las teorías de campos de la ff́sica. El esquema conceptual derivado del epítome 
de la unidad temática se muestra en $\operatorname{los}$ cuadros 2,3 y 4 . Destacamos la necesidad de realizar el mapa conceptual de campos previo a los de ondas y sonido.

Los cuadros 2 y 3 son independientes, stendo el primero el que incluye los conceptos más generales. El cuadro 4 es el que involucra conceptos más concretos.

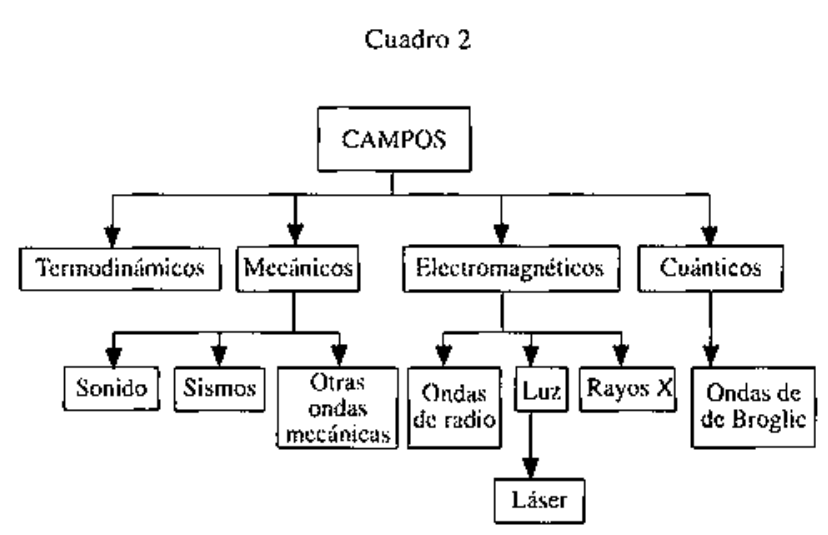

Cuadro 3

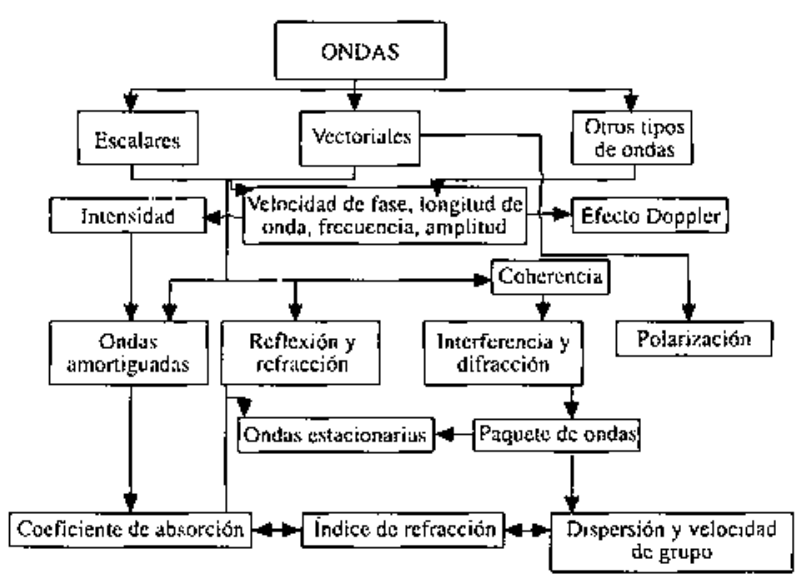

Hemos logrado, de esta manera, establecer las jerarquías conceptuales de acuerdo con la propuesta de Novak (1985) y Moreira $(1985,1987)$.

\section{Objetivos y orientaciones didácticas generales}

La selección de las intenciones, que deben formularse con miras a lograr efectos concretos en el proceso de enseñanza-aprendizaje, es una etapa importante que forma parte de un diseño curricular completo.

Consideramos que la formulación que hemos realizado de los objetivos de aprendizaje va a permitir cambios reales en el comportamiento de los alumnos.

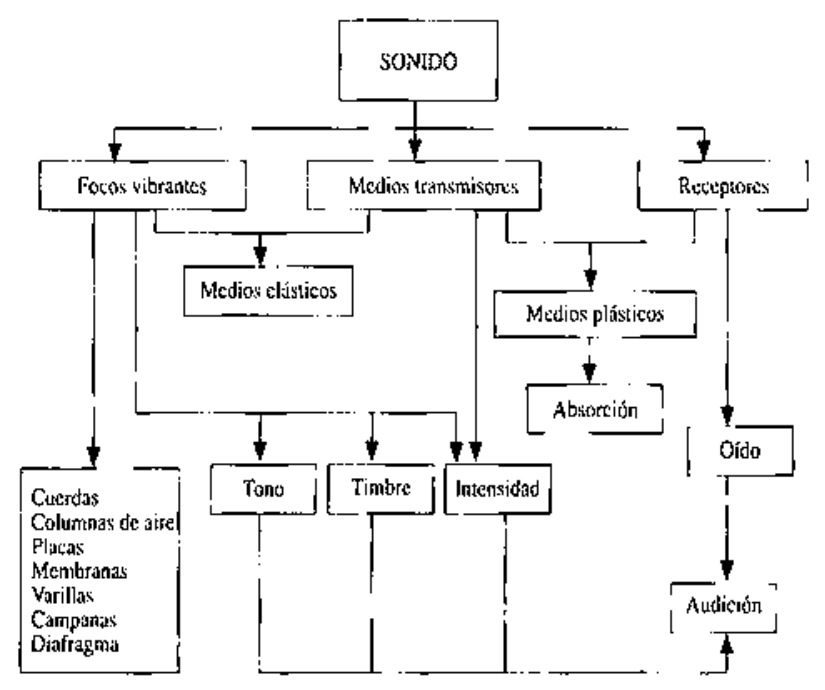

Dichos cambios, encuadrados dentro de la unidad temática elegida, son los que enumeramos a continuación:

\section{Objetivos instruccionales:}

- Describir los principales fenómenos ondulatorios.

- Comprender los conceptos fundamentales que permiten describir dichos fenómenos.

- Relacionar correctamente los conceptos involucrados en las leyes y principios correspondientes.

- Vincular ondas mecánicas con los fenómenos acústi$\cos$.

\section{Objetivos procedimentales:}

- Utilizar criterios adecuados para seleccionar procedi* mientos de aprendizaje referentes a los fenómenos ondulatorios y acústicos.

- Operar en todas las actividades tendientes al logro de los objetivos terminales de la unidad.

\section{Objetivos actitudinales:}

- Demostrar interés, dentro del marco de la unidad, por el desarrollo de valores, normas y actitudes en el com. portamiento.

- Disfrutar con las tareas desarrolladas durante el aprendizaje de los temas involucrados en la unidad.

\section{Contenidos organizadores}

Contemplando lo que prescribe Ia teoría de la elaboración, construimos un cuadro con los hechos, conceptos, 
principios y procedimientos para ondas y sonido. Esto lo realizamos paralelamente a la construcción de los mapas conceptuales.

Estos contenidos organizadores, que son los hechos, conceptos, leyes, etc., vinculados a la temática elegida, proveerán la estructura interna de la organización de contenidos, y para ellos hemos determinado el siguiente esquema clasificatorio (Cuadros 5 y 6 ).

\section{Organización de los contenidos. Niveles de elaboración}

Este diseño tiene un modelo secuencial que consiste en organizar en niveles de elaboración los contenidos.

El primer nivel de elaboración desarrolla contenidos detallados de algunos aspectos del epítome. Este nivel sirve de base para los sucesivos niveles de elaboración.

\begin{tabular}{|c|c|c|c|}
\hline HECHOS & CONCEPTOS & GENERALIZACIONES & LEYFS Y PRINCIPIOS \\
\hline $\begin{array}{l}\text { Cuando se produce una } \\
\text { perturbación en un medio, ésta se } \\
\text { trasmite en forma de onda. } \\
\text { Escuchamos y vemos debido a } \\
\text { fenómenos ondulatorios. } \\
\text { Para producir una onda hay que } \\
\text { realizar un trabajo. } \\
\text { Una onda puede propagarse en l, } \\
2 \text { y } 3 \text { dimensiones. } \\
\text { Cuando a un sistema material } \\
\text { Ilega una onda, éste recibe energia. } \\
\text { Cuando una onda llega a una } \\
\text { superficie de separación de dos } \\
\text { medios con distintas propiedades, } \\
\text { se refleja. } \\
\text { Una onda cambia su dirección de } \\
\text { propagación cuando pasa en forma } \\
\text { oblicua de un medio a otro. } \\
\text { Cuando una onda encuentra un } \\
\text { obstáculo, to rodea. } \\
\text { En ocasiones, cuando dos ondas } \\
\text { se superponen en un lugar, se } \\
\text { anutan o refuerzan su acción. }\end{array}$ & $\begin{array}{l}\text { Onda } \\
\text { Onda escalar } \\
\text { Onda vectorial } \\
\text { Onda mecánica } \\
\text { Onda electromagnética } \\
\text { Onda transversal } \\
\text { Onda longitudinal } \\
\text { Onda estacionaria } \\
\text { Velocidad de propagación } \\
\text { Longitud de onda } \\
\text { Amplitud } \\
\text { Reflexión } \\
\text { Refracción } \\
\text { Índice de refracción } \\
\text { Intensidad } \\
\text { Coeficiente de absorción } \\
\text { Polarización } \\
\text { Interferencia } \\
\text { Difracción } \\
\text { Coherencia } \\
\text { Dispersión } \\
\text { Superposición } \\
\text { Paquete de ondas } \\
\text { Velocidad de grupo } \\
\text { Resonancia }\end{array}$ & $\begin{array}{l}\text { Las ondas transmiten } \\
\text { energía y cantidad de } \\
\text { movimiento lineal y } \\
\text { angular. } \\
\text { Las ondas no transportan } \\
\text { materia. } \\
\text { La velocidad de } \\
\text { propagación de una onda } \\
\text { depende de las } \\
\text { características y del } \\
\text { estado del medio. } \\
\text { La velocidad de una onda } \\
\text { es igual al producto de su } \\
\text { frecuencia y de su } \\
\text { longitud de onda. } \\
\text { Cuando una onda se } \\
\text { refleja sobre una } \\
\text { superficie, el ángulo de } \\
\text { incidencia es igual al } \\
\text { ángulo de reflexión. } \\
\text { La frecuencia de una } \\
\text { onda no se altera cuando } \\
\text { pasa de un medio a otro. } \\
\text { Las ondas transversales } \\
\text { se polarizan. }\end{array}$ & $\begin{array}{l}\text { Leyes del MAS } \\
\text { Leyes de Snell } \\
\text { Ley de Fresnel } \\
\text { Ley de Malus } \\
\text { Principio de Huygens } \\
\text { Principio de conservación } \\
\text { de la energía }\end{array}$ \\
\hline
\end{tabular}

\begin{tabular}{|l|l|}
\hline \multicolumn{1}{|c|}{ PROCEDIMIENTOS } & VALORES, NORMAS, ETC. \\
\hline $\begin{array}{l}\text { Realización de esquemas conceptuales en actividades } \\
\text { Simulación por computadoras } \\
\text { Uso de la V de Gowin } \\
\text { Uso de láminas polaroid } \\
\text { Experimentación con equipos estándar } \\
\text { Consulta bibliográfica } \\
\text { Utilización de dinámicas grupates } \\
\text { Resoltución de situaciones problemáticas referidas al tema }\end{array}$ & $\begin{array}{l}\text { Participación activa en actividades grupales } \\
\text { Respeto a las consignas }\end{array}$ \\
\hline
\end{tabular}


Cuadro 6

Contenidos organizadores de sonido

\begin{tabular}{|c|c|c|c|}
\hline HECHOS & CONCEPTOS & GENERALIZACIONES & LEYES Y PRINCIPIOS \\
\hline $\begin{array}{l}\text { El sonido necesita de un medio } \\
\text { material (sólido, líquido o gas) para } \\
\text { propagarse. } \\
\text { El sonido se origina en cuerdas } \\
\text { vibrantes, columnas de aire vibrante, } \\
\text { placas y membranas vibrantes, etc. } \\
\text { El sonido puede producirse } \\
\text { mediante ondas estacionarias } \\
\text { transversales (instrumentos de } \\
\text { cuerda) o longitudinales } \\
\text { (instrumentos de viento). } \\
\text { Es posible distinguir el sonido } \\
\text { emitido por diferentes instrumentos } \\
\text { musicales. } \\
\text { La altura (frecuencia) de un sonido } \\
\text { (sibbato de un tren) es mayor cuando } \\
\text { la fuente se aproxima a çuien lo oye } \\
\text { que ctando se aleja de El. } \\
\text { La intensidad de un sonido depende } \\
\text { de la distancia del que oye a la } \\
\text { fuente sonora. } \\
\text { La voz se produce por una corriente } \\
\text { de aire expelida por los pulmones } \\
\text { que hace vibrar las cuerdas vocales. } \\
\text { Se oye debido a que el timpano } \\
\text { (membrana) del oído es sensible a } \\
\text { cambios pequeños de presión } \\
\text { producidos por las vibraciones } \\
\text { mecánicas de las ondas sonoras. }\end{array}$ & $\begin{array}{l}\text { Sonido } \\
\text { Intervalo audible } \\
\text { Uitrasonido } \\
\text { Infrasonido } \\
\text { Ruido } \\
\text { Velocidad de propagación } \\
\text { Ondas estacionarias } \\
\text { Frecuencia fundamental } \\
\text { Sobretono } \\
\text { Timbre } \\
\text { Interferencia } \\
\text { Pulsación (batimiento) } \\
\text { Inlensidad } \\
\text { Tono } \\
\text { Nivel de intensidad } \\
\text { Voz } \\
\text { Audición } \\
\text { Resonancia }\end{array}$ & $\begin{array}{l}\text { Las ondas sonoras se } \\
\text { propagan en forma de } \\
\text { ondas mecánicas } \\
\text { Jongitudinales. } \\
\text { La velocidad del sonido } \\
\text { en un medio depende de } \\
\text { las propiedades elásticas } \\
\text { y de la densidad del } \\
\text { mismo. } \\
\text { Las ondas sonoras } \\
\text { trasmiten energía y } \\
\text { cantidad de movimiento } \\
\text { lineal y angular, pero no } \\
\text { materia. } \\
\text { Una onda sonora puede } \\
\text { considerarse como una } \\
\text { onda de desplazamiento o } \\
\text { como una onda de } \\
\text { presión, estando } \\
\text { desfasadas en } 90^{\circ} \text { una } \\
\text { respecto de la otra. } \\
\text { La interferencia entre } \\
\text { ondas sonoras que viajan } \\
\text { en sentidos opuestos } \\
\text { pueden dar lugar a ondas } \\
\text { longitudinales } \\
\text { estacionarias. } \\
\text { El movimiento relativo } \\
\text { entre la fuente y el } \\
\text { observador produce un } \\
\text { cambio aparente en la } \\
\text { frecuencia del sonido. }\end{array}$ & $\begin{array}{l}\text { Conservación de la } \\
\text { energía. }\end{array}$ \\
\hline
\end{tabular}

\begin{tabular}{|l|l|}
\hline \multicolumn{1}{|c|}{ PROCEDIMIENTOS } & \multicolumn{1}{|c|}{ VALORES, NORMAS, ETC. } \\
\hline $\begin{array}{l}\text { Realización de esquemas conceptuales } \\
\text { Experimentación con instrumentos musicales } \\
\begin{array}{l}\text { Consulta bibliográfica } \\
\text { Utilización de dinámicas grupales } \\
\text { Resolución de situaciones problemáticas referidas al tema }\end{array}\end{array}$ & $\begin{array}{l}\text { Participación activa en actividades grupales } \\
\text { Respeto a las consignas } \\
\text { Concienciación acerca de los efectos psicológicos y } \\
\text { fisiológicos del ruido }\end{array}$ \\
\hline
\end{tabular}

Del análisis de las palabras de Coll (1987), a medida que se avanza en Ios niveles de elaboración, aumenta en forma gradual la complejidad con que se abordan los contenidos. Al finalizar cada nivel, debe formularse un epitome ampliado, con una estructuración más compleja.
Los niveles de elaboración, por su parte, proporcionan información cada vez más detallada. Van determinando niveles de complejidad creciente, con la posibilidad de un regreso al panorama inicial globalizado y general (zoom). Así, por ejemplo, un segundo nivel de elabora- 
ción opera sobre los contenidos introducidos en el primer nivel, proporcionando mayores detalles.

Los contenidos se deben organizar con una secuencia lógica entre conceptos, principios, procedimientos y el diseño didáctico para el aprenđizaje.
Es importante observar que nuestra metodología de trabajo permite una elaboración paralela de temas análogos referidos a ondas mecánicas y ondas electromagnéticas, si bien, debido a condicionamientos curriculares, el tratamiento final enfatiza la propagación de las ondas sonoras (Cuadro 7).

Cuadro 7

\section{PRIMER NIVEL DE ELABORACIÓN}

\section{Epitome}

Vivimos rodeados de ondas.

Para comprobarlo basta que miremos el mar o la superficie de un lago. Vemos la televisión, escuchamos la ratio, nos comunicamos telefónicamente o por fax ulilizando fenómenos ondulalorios.

Los terremotos, las cuerdas de los instrumentos musicales y el homo de microondas son ejemplos, entre otros, de propagación ondulatoria.

\begin{tabular}{|c|c|}
\hline Ondas mecánicas & Ondas electromagnéticas \\
\hline Fenómenos ondulatorios: & Fenómenos electromagnéticos: \\
\hline $\begin{array}{l}\text { I.1. Ondas en el agua } \\
\text { I.2. Ondas sísmicas } \\
\text { I.3. Ondas en un resorte } \\
\text { I.4. Ondas sonoras }\end{array}$ & $\begin{array}{l}\text { I.1. Luz } \\
\text { 1.2. Infrarrojo, ultravioleta, ondas de radio, microondas, elc. } \\
\text { I.3. Rayos X }\end{array}$ \\
\hline
\end{tabular}

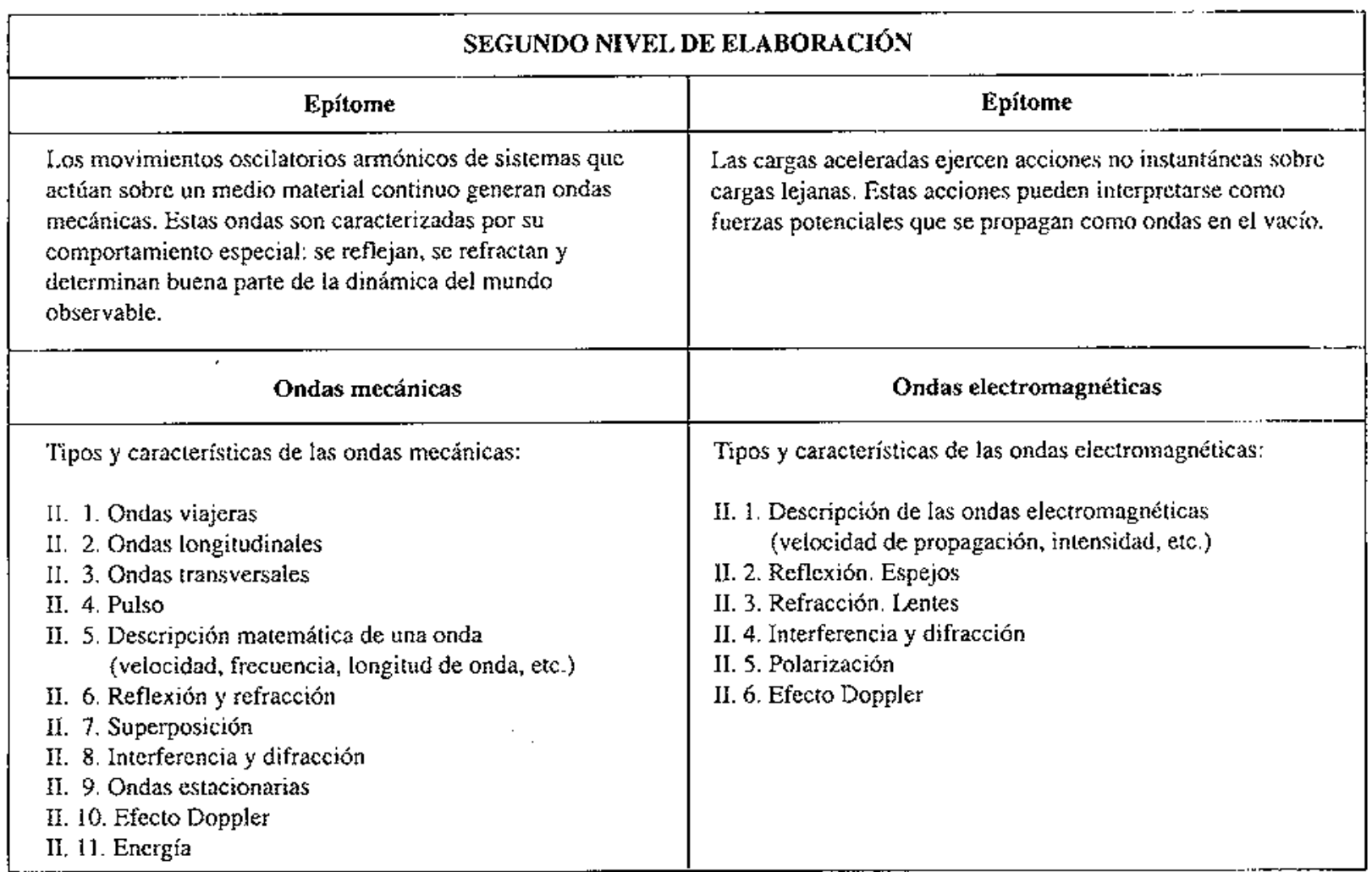




\begin{tabular}{|c|c|}
\hline \multicolumn{2}{|c|}{ TERCER NIVEL DE ELABORACIÓN } \\
\hline Epitome & Epítome \\
\hline $\begin{array}{l}\text { Los sonidos nos proporcionan información de un cierto tipo. } \\
\text { Muchos de ellos son agradables: cl de los instrumentos } \\
\text { musicales, el canto de los pájaros, entre otros. Los hay } \\
\text { desagradibles, que son los que denominamos ruido. } \\
\text { En general, los cuerpos que vibran generan trenes de ondas } \\
\text { en el aire que llegan a nuestros oídos. }\end{array}$ & $\begin{array}{l}\text { Otras informaciones del mundo exterior nos llegan por } \\
\text { medio de las ondas electromagnéticas. Así, por ejemplo, la } \\
\text { Iuz permite al hombre conocer el mundo que lo rodea y } \\
\text { otros tipos de ondas electromagnéticas dan a los científicos } \\
\text { la posibilidad de explorar el universo. } \\
\text { Asociando esto a la tecnología del presente, el panorama se } \\
\text { amplía con el uso de láser, fibras opticas, etc. }\end{array}$ \\
\hline Ondas mecánicas & Ondas electromagnéticas \\
\hline Sonido: & Luz: \\
\hline IFI. 1. Ondas sonoras & III. I. Espectro visibic \\
\hline $\begin{array}{l}\text { 111. 2. Caracteristicas de las ondas sonoras } \\
\text { (tono, timbre e intensidad) }\end{array}$ & $\begin{array}{l}\text { III. 2. Sistemas combinados de espejos y lentes } \\
\text { III. 3. El ojo humano }\end{array}$ \\
\hline III. 3. Instrumentos musicales & III. 4. Instrumentos ópticos \\
\hline IIl. 4. El ó́do y la audición & III. 5. Láser \\
\hline III. 5. Análisis de las ondas sonoras & \\
\hline
\end{tabular}

\section{Evaluación}

La evaluación contempla los siguientes pasos en distintas etapas del proceso:

- Evaluación del diagnóstico: se realiza con un test diseñado en computadora. Los resultados del mismo permiten la detección de los esquemas de conocimiento que tienen los alumnos sobre la unidad a desarrollar, permitiendo los ajustes de las actividades planificadas.

- Evaluación coninua y personatizada de seguimiento: se realiza para el control y ajuste de estrategias desde el punto de vista cognitivo, procedimental y actitudinal.

- Evatuación final con un postest similar al de diagnóstico: su objeto es conocer la evolución o sustitución de los esquemas de conocimiento.

- Evaluación final tradicional: es una prueba que contempla valorar la recreación de conceptos teóricos y de procedimientos de resolución de problemas. Está limitada a Ia evaluación de los conocimientos y al logro de los objetivos instruccionales.

Los tests (pretest y postest) diseñados para computadoras incluyen pantalias de distintos tipos: textos, preguntas de verdadero-falso, preguntas de opciones múltiples, correspondencia entre listas, etc. y presentaciones con animaciones. La variedad de imágenes de estas ullimas, que se intercalan entre las pantallas, es muy amplia. EI tipo de imágenes queda determinado por el objetivo que se quiera lograr con su inclusión, permitiendo reafirmar una respuesta dada por el alumno o ayudarlo en la búsqueda de la respuesta correcta.

\section{APLICACIÓN DEL DISEÑO}

Los resultados obtenidos hasta el momento corresponden a pruebas ejecutadas durante el ciclo lectivo 1994 con ahumnos de nivel universitario básico.

Los ítems temáticos evaluados en un test previo a la aplicación del diseño instruccional (pretest) y en otro posterior a la misma (postest) fueron elegidos según los siguientes criterios:

- se ajustan al diseño curricular elaborado;

- corresponden a contenidos de asimitación considerada dificultosa;

- se adaptan al uso de medios informáticos interactivos (PC) en su implementación;

- se abordan cuestiones, en ambas pruebas, que tienen estructuras conceptuales similares;

- los ítems seleccionados contemplan algunos conceptos generales (asociados a ondas mecánicas) y otros más específicos (asociados a sonido). 
Cuadro 8

\begin{tabular}{|l|l|l|}
\hline \multirow{3}{*}{ ONDAS } & A & Identificar fenómenos onduatorios. \\
\cline { 2 - 4 } & B & Conocer que las ondas mecánicas no transmiten materia sino energía. \\
\cline { 2 - 4 } & C & Analizar las variables que influyen en la frecuencia de vibración de una cuerda tensá. \\
\hline \multirow{3}{*}{ SONIDO } & D & Determinar el mecanismo de producción del sonido. \\
\cline { 2 - 4 } & E & Diferenciar claramente los conceptos de tono, timbre e intensidad de un sonido. \\
\cline { 2 - 4 } & F & Inferir que el sonido necesita de un medio material para propagarse. \\
\cline { 2 - 4 } & G & Analizar el fenómeno de transmisión del sonido en diferentes medios. \\
\cline { 2 - 4 } & H & Distinguir los fenómenos de reflexión, refracción y absorción del sonido. \\
\hline
\end{tabular}

Las pruebas de diagnóstico contemplan los aspectos señalados en el cuadro 8.

El gráfico 1 muestra los resultados obtenidos en los tests propuestos.

Gráfjco 1

Porcentaje de respuestas correctas ( $100 \%$ total de alumnos) para los diferentes ítems del cuadro 4.

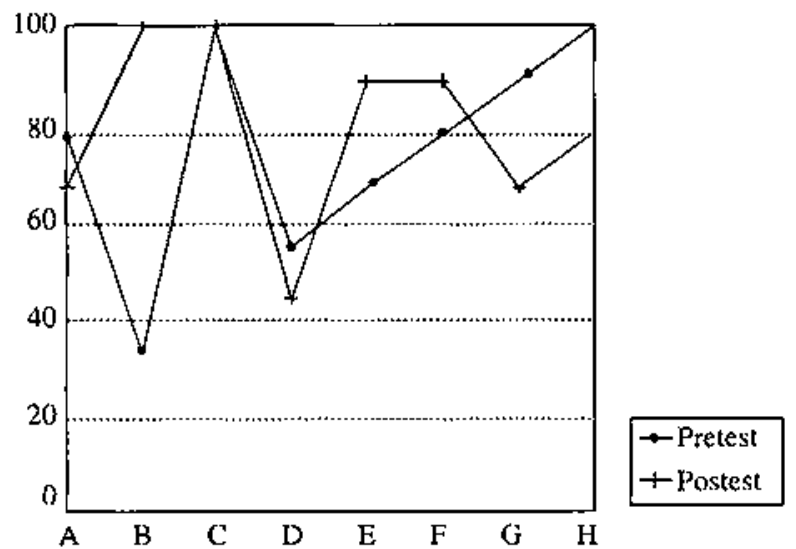

Con el objeto de evitar el «recuerdo» del pretest, como postest se utilizó una prueba levemente diferente, aunque ambos tests fueron referidos a la exploracion de los mismos conceptos.

A partir del gráfico l podemos observar que, en los ítemes A, D, G y H, los resultados del diseño instruccional resultaron poco satisfactorios. Analizados los mismos, consideramos que éstos se deben a ciertas difi- cultades planteadas desde el punto de vista de esquemas de conocimiento precientíficos de los alumnos $y$, en menor medida, de "fluctuaciones estadísticas» no muy marcadas.

Hemos detectado a través de apreciaciones cualitativas que las dificultades puestas de manifiesto en el postest, que no habían aparecido anteriormente en el pretest, fueron las siguientes (de acuerdo con el cuadro 8):

Ítem A: Una (de cuatro) de las opciones de la situación planteada en el postest demuestra que los alumnos confunden entre los sentidos de olfato y audición (algunos estudiantes consideraron el aroma como un fenomeno ondulatorio).

Ítem D: En el pretest se pide identificar Ja producción del sonido en un ejemplo muy relacionado con la vida diaria como es la voz. En cambio, en el postest, se recurre a instrumentos musicales, de los que los alumnos no tienen tanto conocimiento empírico.

Ítem G: En este caso podemos hablar de una variación de la dificultad de la cuestión planteada, ya que en el pretest la señal se propaga en un único medio y en el postest se introduce un cambio de medio de propagación, detectándose falta de dominio en la interpretación de esto último.

Ítem $H$ : En el pretest se da un ejemplo muy difundido (fenómeno del eco en una montaña). En cambio, en el postest, se recurre al análisis de una situación menos frecuente (como es hablar dentro de una cueva).

Por otra parte, Jas ideas previas de los alumnos fueron modificadas durante el proceso en forma favorable, logrândose porcentajes bastante altos en el postest, en los ítems B, C, E y F. Podemos comprobar que en algunos ítems se mantuvieron los resultados y en otros se 
superaron, en especial en lo que se refiere a: las formas de produccíón del sonido, las características del mismo y también a que las ondas mecánicas necesitan de un medio material para su propagación.

Ambos tests se confrontaron con una prueba tradicional de resolución de preguntas teóricas y problemas, esta última formando parte de los requerimientos de evaluación para promoción en el curso. En la prueba tradicional se obtuvieron, en general, buenos resultados. Dadas las características de esta evaluación, no se puede confrontarla con el postest, pero se puede vincular con los resultados de las otras evaluaciones que se hicieron a lo largo del curso. Los resultados figuran en el gráfico 2 .

\section{Gráfico 2}

Porcentaje de alumnos aprobados en función de los intervalos de puntaje obtenido (máximo 100 puntos).

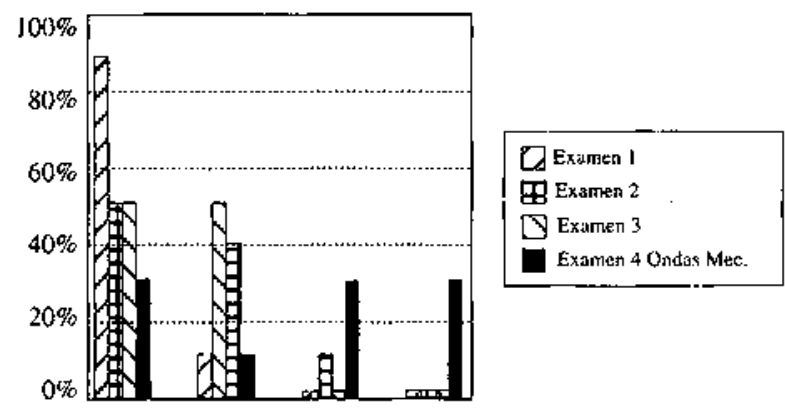

Los resultados comparativos muestran que hay una superación en el examen referido a ondas mecánicas y sonido, con respecto a los anteriores.

Cabe añadir que la evaluación continua de seguimiento de los alumnos, de tipo cualitativa, confirma la necesidad de estrategias variadas.

\section{CONCLUSIONES}

El diseño curricular ha permitido concretar dos etapas importantes, una referida al trabajo del equipo de investigación y la otra, a la aplicación y evaluación de la acción en el proceso de enseñanza-aprendizaje.

Ambas instancias son valiosas, dado que, por un lado, ha significado una labor intelectual creadora para el equipo de trabajo, con una profundización de los conocimientos derivados de la investigación educativa y, por otra parte ha posibilitado realizar una acción de transferencia de todo este bagaje enriquecedor al aula.

Este diseño tiene una coherencia en su estructura tal que permite que el alumno asimile gradualmente a través de conceptos, procedimientos y principios. Sin embargo, parece subsistir, como duda, si realmente todos los aprendizajes han sido significativos.

La estructura de la unidad da resultados positivos al evaluar el aprendizaje con la forma tradicional, aunque en menor grado cuando se evalúa con nuevas técnicas (vía análisis de esquemas de conocimiento en computadora personal).

Si atendemos la opinión de los investigadores, encontramos que Pérez Gómez (1989), al referirse a este tipo de diseño curricular, expresa que tiene un fuerte apoyo en la psicología cognitiva y un coherente desarrollo interno, pero que este diseño condiciona profundamente los métodos de enseñanza, siendo lo más adecuado, en este contexto, la exposición verbal. A tal efecto, disentimos con el autor antes mencionado, en base a nuestra experiencia personal como docentes y a la evaluaciones de seguimiento realizadas. Es por esto que tratamos, durante la experiencia, de aplicar otras metodologías con una participación activa de los alumnos en estrategias motivadoras y contributivas. Además, buscamos en todo momento que no predominaran los contenidos sobre los procesos.

Gimeno Sacristán (1994), por su parte, caracteriza el modelo de Coll como el de un esquema globalizador y determina que es aplicable a contenidos concretos que tengan una estructura muy clara, definible y unívoca. Dado que la estructura de los contenidos de física cum. ple con estas condiciones, consideramos que nuestra experiencia es altamente positiva.

Queda analizar si este diseño se puede utilizar dentro de un planeamiento total de la asignatura para que produzca verdaderos cambios significativos en los aprendizajes.

\section{NOTA}

Una versión resumida de este trabajo se presentó en la Novena Reunión Nacional de la Fístca- REF9 (Salta, Argentina, 1995).

\section{AGRADECIMIENTO}

El presente trabajo ha sido realizado con el apoyo de la Universidad Nacional de San Juan (Argentina), que avaló y subsidió las investigaciones realizadas. 


\section{REFERENCIAS BIBI.IOGRÁFICAS}

ALVARENGA, B. y MAXIMO, A. (1983). Física general (con experimentos sencillos). México: Editorial Harla.

COLI., C. (1987). Psicología y curriculum. México: Editorial Paidós Mexicana.

COLL, C. (1991). Aprendizaje escolar y construcción del conocimiento. México: Editorial Patdós.

GETTYS, W.E. et al (1991). Física clásica y moderna. España: Editorial McGraw Hill.

GIMFNO SACRISTÁN y PÉREZ GÓMEZ (1989) Teoría y práctica de la enseñanza. España: Editorial Akal.

GIMENO SACRISTÁN y PÉREZ GÓMEZ (1994). Comprendery transformar la enseñanza. España: Ediciones Morata, SL.
GUTIÉRRF7, R. (1992). La teoría de la elaboración en la práctica. Gúa de trabajo para el diseño curricular. Monografía núm. 1 l. Documentos del i.e.p.s.

MOREIRA, M.A. (1985). Atividade docente na universidade: alternativas instrucionais. Porto Alegre, Brasil: D.C. Luzzato, Ed. da FURG.

MOREIRA, M.A. y BUCHWEITZ, B.(1987). Mapus conceituais. Instrumentos didáticos, de avaliaçâo e de análise de currículo. Brasit: Editora Moraes.

NOVAK, J. (1985). Teoría y práctica de la educación. España: Alianza.

RESNICK, R. y HALLIDAY, D. (1980). Fisica. Parte 1. México: Editorial CECSA.

SEARS, ZEMANSKY y YOUNG (1988). Fisica universitaria. Estados Unidos: Editoriat Addison-Wesiey Iberoamericana.

[Artículo recibido en enero de 1996 y aceptado en octubre de 1996.] 